\title{
High-resolution EEG techniques for brain-computer interface applications
}

\author{
Febo Cincotti $^{\mathrm{a}}$, Donatella Mattia ${ }^{\mathrm{a}}$, Fabio Aloise ${ }^{\mathrm{a}}$, Simona Bufalari ${ }^{\mathrm{a}}$, \\ Laura Astolfi $^{\text {a,b,c }}$, Fabrizio De Vico Fallani ${ }^{\text {a,d }}{ }^{\text {, Andrea Tocci }}{ }^{\text {a }}$, Luigi Bianchi ${ }^{\text {a,e }}$, \\ Maria Grazia Marciani ${ }^{\text {a,e }}$, Shangkai Gao ${ }^{\mathrm{f}}$, Jose Millan ${ }^{\mathrm{g}}$, Fabio Babiloni ${ }^{\text {a,b,* }}$ \\ a IRCCS “Fondazione Santa Lucia”, Rome, Italy \\ ${ }^{\mathrm{b}}$ Department of Human Physiology and Pharmacology, University "La Sapienza”, Rome, Italy \\ c Dep. Informatica e Sistemistica, University "La Sapienza”, Rome, Italy \\ d Interdep. Research Centre for Models and Information Analysis in Biomedical Systems, University "La Sapienza”, Rome, Italy \\ e Department of Neuroscience, University of Rome "Tor Vergata", Rome, Italy \\ ${ }^{\mathrm{f}}$ Department of Biomedical Engineering, Tsinghua University, Beijing, China \\ g IDIAP Research Institute, Martigny, Switzerland
}

Received 29 November 2006; received in revised form 29 June 2007; accepted 29 June 2007

\begin{abstract}
High-resolution electroencephalographic (HREEG) techniques allow estimation of cortical activity based on non-invasive scalp potential measurements, using appropriate models of volume conduction and of neuroelectrical sources. In this study we propose an application of this body of technologies, originally developed to obtain functional images of the brain's electrical activity, in the context of brain-computer interfaces (BCI).

Our working hypothesis predicted that, since HREEG pre-processing removes spatial correlation introduced by current conduction in the head structures, by providing the BCI with waveforms that are mostly due to the unmixed activity of a small cortical region, a more reliable classification would be obtained, at least when the activity to detect has a limited generator, which is the case in motor related tasks.

HREEG techniques employed in this study rely on (i) individual head models derived from anatomical magnetic resonance images, (ii) distributed source model, composed of a layer of current dipoles, geometrically constrained to the cortical mantle, (iii) depth-weighted minimum $\mathrm{L}_{2}$-norm constraint and Tikhonov regularization for linear inverse problem solution and (iv) estimation of electrical activity in cortical regions of interest corresponding to relevant Brodmann areas.

Six subjects were trained to learn self modulation of sensorimotor EEG rhythms, related to the imagination of limb movements. Off-line EEG data was used to estimate waveforms of cortical activity (cortical current density, CCD) on selected regions of interest. CCD waveforms were fed into the BCI computational pipeline as an alternative to raw EEG signals; spectral features are evaluated through statistical tests $\left(r^{2}\right.$ analysis), to quantify their reliability for BCI control. These results are compared, within subjects, to analogous results obtained without HREEG techniques. The processing procedure was designed in such a way that computations could be split into a setup phase (which includes most of the computational burden) and the actual EEG processing phase, which was limited to a single matrix multiplication. This separation allowed to make the procedure suitable for on-line utilization, and a pilot experiment was performed.

Results show that lateralization of electrical activity, which is expected to be contralateral to the imagined movement, is more evident on the estimated CCDs than in the scalp potentials. CCDs produce a pattern of relevant spectral features that is more spatially focused, and has a higher statistical significance (EEG: $0.20 \pm 0.114$ S.D.; CCD: $0.55 \pm 0.16$ S.D.; $p=10^{-5}$ ). A pilot experiment showed that a trained subject could utilize voluntary modulation of estimated CCDs for accurate (eight targets) on-line control of a cursor.

This study showed that it is practically feasible to utilize HREEG techniques for on-line operation of a BCI system; off-line analysis suggests that accuracy of BCI control is enhanced by the proposed method.
\end{abstract}

(C) 2007 Elsevier B.V. All rights reserved.

Keywords: High-resolution electroencephalography; EEG-based brain-computer interfaces; Assistive technologies; Neuroimaging; Motor cortex

\footnotetext{
* Corresponding author at: Department of Human Physiology and Pharmacology, University of Rome "La Sapienza", P.le A. Moro 5, 00185 Rome, Italy. Tel.: +39 328 7697914; fax: +390649910917.

E-mail address: Fabio.Babiloni@uniroma1.it (F. Babiloni).
} 


\section{Introduction}

\subsection{High-resolution EEG}

Electroencephalographic (EEG) recordings have helped clinicians for decades to diagnose specific diseases of the central nervous system. On the other side, its use as a neuroimaging tool has been limited for a long time by the low technology of the system (a few electrodes placed on the scalp of the subject that allow plotting traces of the electrical potentials time course), which did not allow a full exploitation of its potentialities.

The ultimate problem of a neuroimaging technique is the detection of the pattern of activation of cerebral structures in a given experimental condition. In this perspective, the scalp EEG potential traces, or even the scalp potential maps, can only be regarded as a partial result, since they do not carry direct information on the cerebral processing. In the last 20 years, the development of recording and analysis techniques has substantially increased the quality of the EEG recordings. Such an advanced body of techniques, named high-resolution EEG (HREEG)(Babiloni et al., 2004a,b; He et al., 2006; Nunez, 1995; Urbano et al., 1998), has provided an affordable neuroimaging tool with complementary features to other techniques based on metabolic or hemodynamic processes of the brain (Babiloni et al., 2005). The aim of HREEG is to provide standard and tested algorithms that help the experimenter to infer the nature of cerebral activity from the measured scalp potentials. This goal is achieved, for instance, through spatial filtering, which counteracts the smearing of scalp potentials introduced when ionic currents cross low conductivity structures (i.e., the skull). A simple and typical spatial filtering algorithm is the Surface Laplacian (SL) (Nunez, 1995). The estimation of the SL may assume standard electrode locations (e.g. 10-20 system) and simple geometries as a model of scalp shape, such as a plane or a sphere. Nevertheless, the use of digitized electrode locations together with realistic geometries of subject's head, such as those derived from magnetic resonance images (MRIs), has shown to be more accurate (Babiloni et al., 2002, 2004a,b), although more complex to implement.

When it is important to identify the neuroelectrical sources of cortical activity (registered to brain anatomy), the real shape of all structures of the head must be taken into account, together with their conductivity properties. Several approaches are available, each differing from the other in the hypotheses assumed for several key aspects of the solution: size and number of the sources, distribution and orientation with respect to the surface of the cerebral cortex, constraints on the energy of the solution, etc. (for a review see Michel et al., 2004). For instance a method, called ELECTRA (Grave de Peralta et al., 2000), estimates local field potentials, i.e., providing information analogous to intracranial recordings. It is worth to note that, apart from ELECTRA, no other existing method allows to estimate local field potentials from scalp EEG. Other methods estimate the punctual (equivalent current dipoles, ECD) or distributed extracellular currents (cortical current density, CCD) flowing close to the active neuronal populations. The latter methods better model those situations in which a distributed cortical network is supposed to be active (Babiloni et al., 2005; Dale and Sereno, 1993; Dale et al., 2000). This approach also implies the use of realistic head models, reconstructed from MRIs, as volume conductor medium. The accuracy of inverse solution depends on the proper selection of several factors:

1. Appropriate source model; it includes all brain structures that plausibly contributed to the generation of the measured potentials; this is accomplished by discretizing the whole cerebral cortex with thousands of equivalent current dipoles.

2. Accurate volume conductor model; it describes the electrical properties of the head that influence the spread of ionic currents from the cortex to the scalp (the so-called forward problem). Using anatomic MRI of individual subjects, a three-shell boundary element model (BEM) of the head describing four compartments of the model (brain, skull, scalp, and air) is derived and used to compute a lead field matrix. It is worth to note that although finite element modeling (FEM) could allow a finer grained model of the volume conductor space, the lack of reliable information about conductivity values degrades accuracy of FEM solutions, yet requiring a higher computational effort than BEM. Hence, in this study the forward problem will be solved using a three shell BEM approach.

3. Accurate determination of electrode positions; digitization of actual electrode three-dimensional positions accounts for misplacement of sensors with respect to standard positions (e.g. the standard 10-20 configuration).

4. A priori constraints. A unique solution can only be obtained by introducing additional constraints to solve an otherwise under-determined inverse problem. Constraints on minimum energy of solution (Dale and Sereno, 1993; Hämäläinen and Ilmoniemi, 1984) are usual, but more specific information on the nature of noise or signal can be introduced using metric matrices (see Eq. (2)).

\subsection{Brain-computer interfaces}

Brain-computer interfaces (BCIs) can provide non-muscular communication and control for people with severe motor disabilities (Wolpaw and Birbaumer, 2006). This can be obtained by extracting information about user's will directly from signals originating in the central nervous system. Current BCIs use a variety of invasive and non-invasive methods to record brain signals and a variety of signal processing methods (for a review, see Birbaumer and Cohen, 2007; Wolpaw, 2007). Interest on the application of advanced EEG neuroimaging procedures in the context of the BCI derives from two main factors: (i) the refinement of recording and analysis methods is likely to improve performance in the $\mathrm{BCI}$ operation; ii) the advantages of the HREEG technology can be exploited to improve the understanding of the some of the processes which underlie the subjects' "BCI-control" capabilities.

It has been previously reported that spatial filtering procedures (including the computation of surface Laplacian) improve reliability of a BCI device (McFarland et al., 1997). In fact, spatial filtering applied to the raw EEG potentials reduces the 


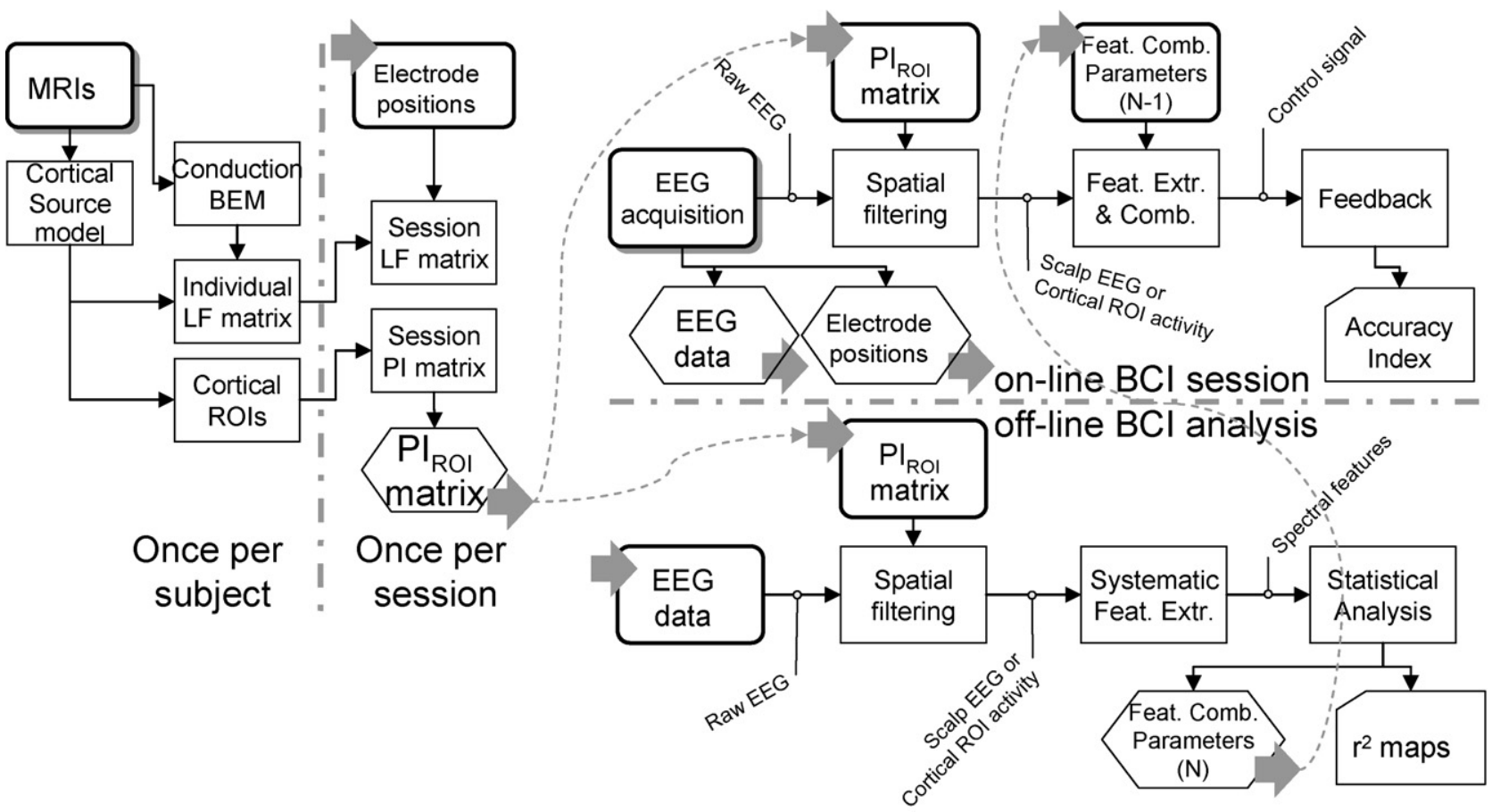

Fig. 1. Block diagram of the approach proposed for integration of high-resolution EEG technique into BCI processing. Acronyms: MRI: magnetic resonance images; BEM: boundary element model; LF: lead field; ROI: region of interest; PI: pseudo-inverse; EEG: electroencephalography; Feat. Extr., Comb.: feature extraction, combination.

correlation between recording channels (due to current spread in the head volume conductor). Spatially filtered potentials are thus more related to cortical sources located under each electrode, allowing following processing to rely on more distinct spatial patterns. The use of preprocessing methods based on biophysical consideration, allows the use of simpler processing modules. This is valuable for any BCI that (i) exploits EEG features generated by localized cortical sources and (ii) relies on simple feature classifiers. The latter point is a key issue in the subject's training phase, since it allows the subject identify a simple relationship between the feedback he/she receives and the modifications of EEG signals he is learning to self induce.

In this respect, cortical source estimation is likely to provide a better signal conditioning in preparation of BCI processing. In fact, it is well know that the estimation of the cortical activity from scalp EEG data by solving the linear inverse problem with realistic head models greatly enhances the spatial details available when compared to the Surface Laplacian methods (Babiloni et al., 2000; Cincotti et al., 2004a; He et al., 2006; Mattia et al., 2006; Nunez, 1995; Urbano et al., 1998).

The aim of the present work is then to describe the chain of signal processing methods that have been found to improve accuracy of an EEG-based BCI system, operated by modulation of the sensorimotor rhythms induced by motor imagery tasks. In the approach followed here, cortical regions of interest are segmented according to the individual brain anatomy to represent task-relevant patches of motor cortex. The signal conditioning procedure allowed quantifying the time-varying electrophysiological activity (i.e., CCD) of these selected cor- tical regions using non-invasive HREEG recordings. Spectral features derived from CCDs and from scalp potentials are first compared in terms of discrimination capability (statistical mapping) and then in terms of correct classification rate in a simple binary selection task, both off- and on-line.

A block diagram showing the steps described in the Section 2 is given in Fig. 1

\section{Methods}

\subsection{Subjects and EEG data acquisition}

Six subjects (males; mean age $30.2 \pm 2.9$ ) voluntarily participated to the study. One of the six subjects (S5) presented a traumatic stabilized lesion located at the dorsal level and he was confined to a wheelchair. Written informed consent was obtained from each subject, after explanation of the study, approved by the local ethics committee. All subjects were right-handed as assessed by the Edinburgh inventory, while for the disabled subject this was based on a personal interview.

Subjects underwent a series of EEG acquisition sessions, in which they were trained to gain control of their sensorimotor rhythms (mu-rhythm) to operate a brain-computer interface (BCI) system. Each session lasted about $40 \mathrm{~min}$ and consists of eight 3-min runs of 30 trials each. We collected a total of 8-12 training sessions for each subject; training ended when performance was stabilized.

During acquisition, subjects were comfortably seated on a reclining chair (or when necessary a wheelchair) in a dimly 
lit room. Scalp potentials were collected from 59 positions (according to an extension of the 10-20 International System) and amplified by a commercial EEG system (BrainAmp, Brainproducts $\mathrm{GmbH}$, Germany). EEG signal was bandpass filtered between 0.1 and $70 \mathrm{~Hz}$, digitized at 200 samples/s and transmitted to the BCI program for on-line processing. EEG data were also stored for off-line analysis.

Subjects were instructed to minimize muscular, electrooculographic, and blink activity during the active modulation phase of trials. A technician, who monitored the ongoing EEG traces, reminded the subject those instructions at each occurrence of such an artifact, so that on-line performance was minimally affected by artifacts. For the purpose off-line analysis, EEG traces were reviewed by an expert electroencephalographer, and EEG segments containing artifacts were rejected.

\subsection{Experimental task}

Each trial consisted of four phases:

1. Target appearance: a rectangular target appeared on the right side of the screen, covering either the upper or the lower half of the side.

2. Feedback phase: $1 \mathrm{~s}$ after the target, a cursor appeared in the middle of the left side of the screen and moved at a constant horizontal speed to the right. Vertical speed was determined by the amplitude of sensorimotor rhythms (see Section 2.4). A cursor sweep lasted about $3 \mathrm{~s}$.

3. Reward phase. If the cursor successfully hit the target, the latter flashed for about $1 \mathrm{~s}$. Otherwise, it just disappeared.

4. Intertrial interval. The screen stayed blank for about $2 \mathrm{~s}$, in which the subject was allowed to blink and swallow.

Fig. 2 shows the feedback screen used in the BCI training sessions.

Subjects were aware that the increase or decrease of a specific rhythm in their EEG produced a movement of the cursor towards the top or the bottom of the screen. They were suggested to concentrate on kinesthetic imagination of upper limb movements

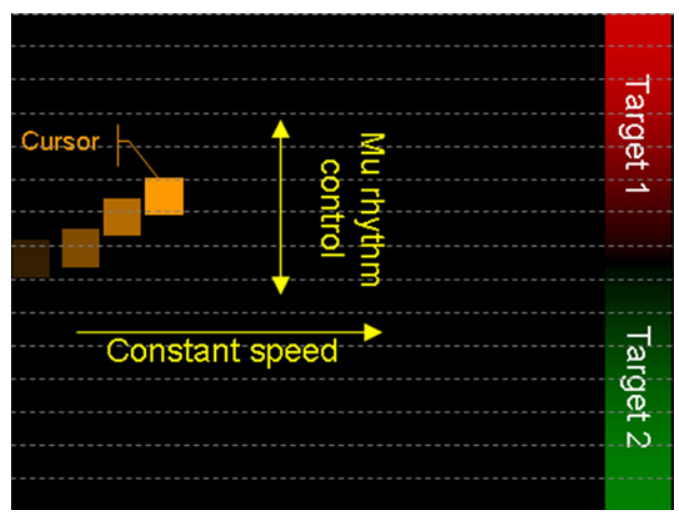

Fig. 2. Feedback screen used in the BCI training sessions. After a target appeared on the right side of the screen, a cursor sweeps horizontally, while its vertical position was defined by the amplitude of sensorimotor EEG rhythms that the subject is learning to regulate. (e.g. fist clenching) to produce a desynchronization of the murhythm on relevant channels (cursor up), and to concentrate on kinesthetic imagination of lower limb movements (e.g. repeated dorsiflexion of ankle joint) to produce a contrasting pattern (with possible desynchronization of mu/beta-rhythm over the mesial channels, cursor down).

Using this simple binary task as performance measure, training is meant to improve performances from 50 to $70 \%$ to 80 to $100 \%$ of correct hits.

\subsection{On-line processing}

Digitized EEG data were transmitted in real time to the BCI2000 software system (Schalk et al., 2004), which performs performed all necessary signal processing and displayed feedback to the user. The processing pipe can be considered of several stages, which process the signal in sequence. Only the main ones will be mentioned below: spatial filter, spectral feature extraction, feature combination, and normalization.

\subsubsection{Spatial filter}

A general linear combination of data channels is implemented by defining a matrix of weights that is multiplied to each time sample of potentials (vector). This allowed implementation of different spatial filters, e.g.: (i) re-referencing to common average (CAR), which was used during the training phase, and (ii) estimation of CCD waveforms on cortical ROIs, using weights derived in Section 2.8 .

\subsubsection{Spectral feature extraction}

Spectral feature extraction was performed every $40 \mathrm{~ms}$, using the latest $300 \mathrm{~ms}$ of data. An autoregressive spectral estimator, based on the maximum entropy algorithm, yielded an amplitude spectrum with resolution of $2 \mathrm{~Hz}$. Maximum frequency was limited to $60 \mathrm{~Hz}$

\subsubsection{Feature selection and combination}

A small subset of those spectral features (frequency bins $\times$ EEG channels) that were significantly modulated by the motor imagery tasks were linearly combined to form a single control signal. Selection of responsive channels and frequency bins, and determination of combination weights were operated before each on-line session (see Section 2.4). In general, only two or three spectral amplitude values (depending on individual patterns) were generally used to obtain the control signal.

\subsubsection{Normalization}

The control channel was detrended to avoid biases of the cursor, and scaled so that the resulting vertical deflection of the feedback cursor was visible but not saturated. In fact, the vertical position of the cursor was updated every $40 \mathrm{~ms}$ by a number of pixels (positive or negative) equal to the output by this stage. Normalization was adaptive, and based on the estimate of the moving average and standard deviation of the control signal.

During the very first session of each subject (screening session), since no off-line analysis was available to guide feature 
selection and combination, the subject was given no on-line feedback (targets only).

\subsection{Off-line analysis}

After artifact rejection, the EEG interval corresponding to the feedback phase were binned into two classes - up or down, depending on the target appeared in each trial.

The spatial filtering and feature extraction stages of the online processing were replicated. Since no feedback delay issue had to be considered during the off-line analysis, spectral estimation was computed on $1 \mathrm{~s}$ long epochs, overlapped by $50 \%$ (i.e., only five spectral estimates had to be computed for each $3 \mathrm{~s}$ long trial, yielding about 600 spectral estimates per class for the whole session).

For each of the 59 channels and 30 frequency bins (1770 features), a contrast was performed, to assess statistically significant modulations induced on a specific feature. To this aim, we computed for each feature (dependent variable) the coefficient of determination $\left(r^{2}\right)$ i.e., the proportion of the total variance of the feature samples accounted for by target position. This index had been previously utilized in literature for similar experimental setups (McFarland et al., 1997; Wolpaw and McFarland, 2004; Wolpaw et al., 2002), and allows direct comparison with published results. A fictitious independent variable was created, using values +1 or -1 in correspondence of "down" or "up" epochs, respectively. A negative sign was attributed to the $r^{2}$ value when dependent and independent variables were controvariant. Viewing statistical results from a different point of view, features characterized by a high $r^{2}$ value are those that maximize prediction of the current target.
Higher values of $r^{2}$ indicate that the subject has gained steadier control of EEG rhythms (in fact they generally increase during the training, from values below 0.1 to values above 0.3 ).

A graphical representation of statistical analysis is shown in Fig. 3, left panel. Each row corresponds to a different EEG channel, columns relate to different frequency bins. Single columns can be interpolated on a topographic arrangement, showing the spatial patterns of significance at a specific frequency (Fig. 3, right panels).

After each training session, $r^{2}$ matrices and maps are computed to determine those significant features that will be used in the Feature selection and combination stage of the next online session. While there might be variations due to less clear patterns, two features coming from channels over the lateral sensorimotor cortices, and one coming from a mesial channel where selected when possible. They were given a weight equal to +1 or -1 , according to the co- or contro-variant behavior of the feature.

\subsection{Lead field matrix}

Each participant underwent a Magnetic Resonance Image (MRI) of the brain to allow the modeling of the head structures. Magnetization prepared rapid gradient echo (MPRAGE) T1 weighted images $\left(256 \times 256\right.$ matrix, 182 slices, $1 \mathrm{~mm}^{3}$ isotropic voxels, repetition time $11.4 \mathrm{~ms}$, echo time $4.4 \mathrm{~ms}$, flip angle $=15^{\circ}$ ) were obtained with a Siemens $1.5 \mathrm{~T}$ Vision Magnetom MR system (Germany).

MRIs of each subject were processed using the Curry 4.6 software (Compumedics Neuroscan Ltd, El Paso, TX), which was also used to compute the lead field matrix.

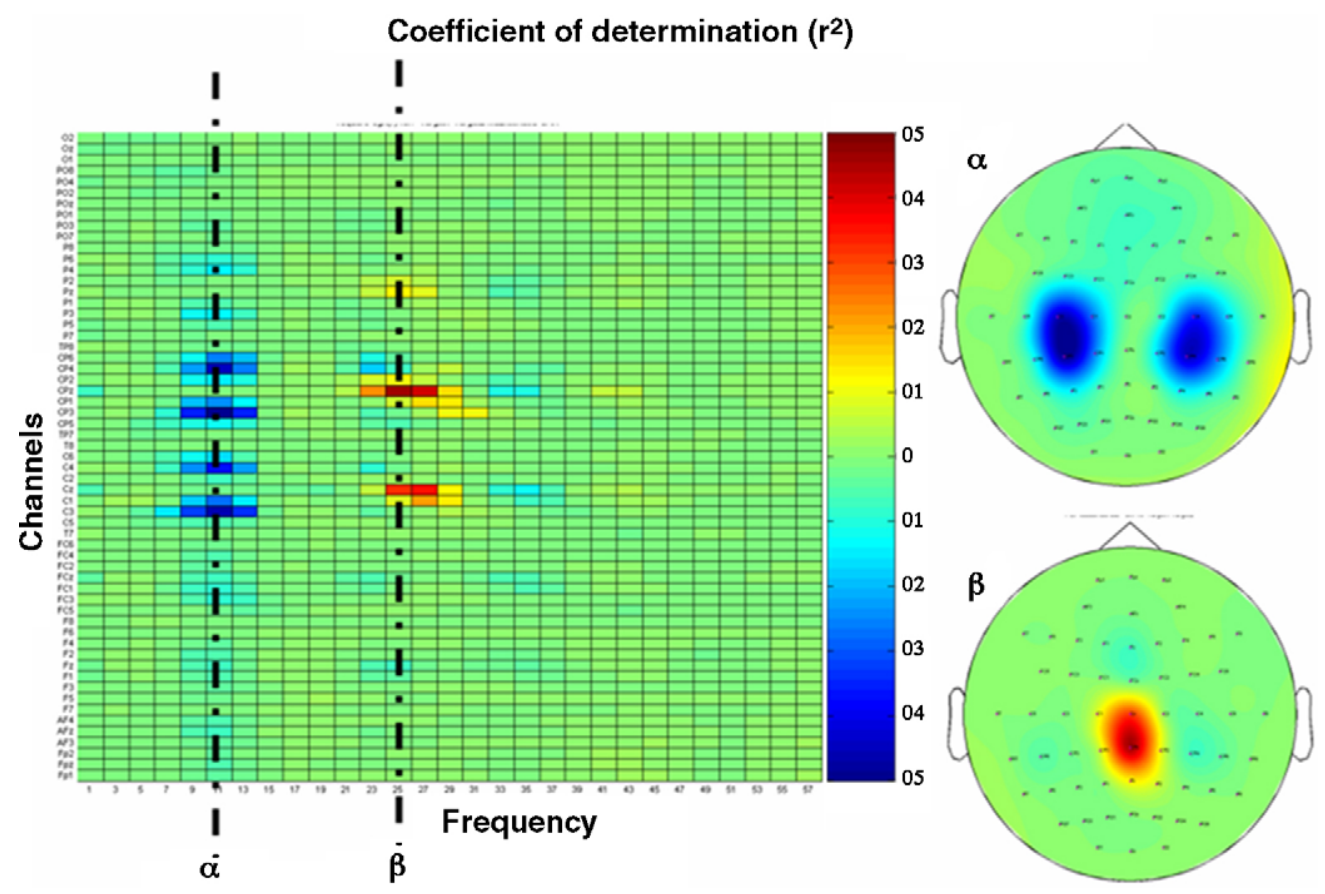

Fig. 3. Graphical representation of the systematic statistical comparison $\left(r^{2}\right.$ value $)$ of each EEG feature extracted by scalp potentials. Left panel: each row of the matrix corresponds to a different EEG channel, columns relate to different frequency bins. Right panel: topographical mapping of $r^{2}$ values, obtained by interpolation of single columns of the matrix; spatial patterns of significance are shown in the alpha and beta band. 
Images were stacked into a 3D isotropic volume and regions containing different tissues were segmented (white matter, grey matter, CSF, skull, scalp and air). Three craniometric repere points (nasion, left and right preauricular points) were marked to allow later registration with other geometrical data. Three tessellated surfaces were extracted (inner and outer skull surface and scalp surface, about 500 triangles each) and used to define the geometry of the BEM. Conductivity of the skull compartment was set to $15 \mathrm{mS} / \mathrm{m}$; conductivity of both the scalp and the brain compartments was set 15 times higher than the skull (Oostendorp et al., 2000).

The cortical sources were modeled by using a distributed model with realistic cortical shape (Babiloni et al., 2000, 2005). A tessellated surface representing the cortical mantle (about 6000 triangles) was extracted through an iterative procedure, which fitted it halfway between the white/gray and the gray/CFS interfaces. The source model was composed of about 3000 current dipoles, positioned at the vertices of the cortical tessellation, with direction normal to the local pseudo-tangent plane. With this approach, the relevant geometric features are preserved and the orientation of each dipole is constrained to be perpendicular to the cortical mantle, thus modeling the alignment of the pyramidal neurons. The actual strength of these sources is later estimated by using a linear inverse procedure according to a weighted-minimum norm approach (see below).

Fig. 4, left panel, shows the typical geometry of the three shell BEM and of the source model.

A "fine-grained" lead field matrix was finally computed, describing the influence of each source on the potential "virtually" measured at each of the about 500 vertices of the scalp mesh. The actual lead field, relative to the measured sensor configuration, will only be computed in a second step, by interpolation from the fine-grained one.

The whole procedure, which is relatively time consuming, was performed only once per subject, as soon as the MRI are available, and in advance with respect to the first EEG recording. Only the lead-field interpolation (which involves a negligible computational effort) had to be repeated for each EEG acquisition session, since it depend on the actual position of electrodes.

\subsection{Region of interest}

Cortical regions of interest (ROIs) were drawn on the tessellated model of the cortical mantle, for each subject. As for the frontal medial wall motor areas, namely the supplementary motor area proper (SMAp) and the caudal cingulate motor area (CMAc) (Picard and Strick, 1996), we followed the anatomical landmarks and defined the anterior border of the SMAp and the CMAc by a plane perpendicular to the anterior-posterior commissure (AC-PC) line at the level of the AC (VAC). The posterior border of both SMAp and CMAc was at a perpendicular plane at the posterior commissure (VPC). The border between the SMAp and CMAc was settled either right above the cingulate sulcus, or in case of a prominent paracingulate sulcus, above it. The primary motor foot (MI-foot) representational area was defined arbitrarily as a region ranging anteriorly to posteriorly from the VAC to the first two thirds of the paracentral lobule and inferiorly bordered by the superior bank of the cingulate sulcus. This region should plausibly include the foot representation site within the MI according to its somatotopic organization. To define the primary motor hand and lip representational area, we followed an anatomical-functional procedure previously reported (Mattia et al., 2006). The anatomical localization of other EEG sources were related to the major sulci and gyri distinguishable on the individual MRIs, and named according to Brodmann nomenclature BA6, BA5, BA7, BA8 and BA9/46).

The segmentation procedure yielded a set of indices of the source dipoles comprised in each ROI. This procedure was performed only once per subject, as soon as the cortical model was available.

Indices of sources belonging to each ROI have been used after estimation of CCD at each dipole, to obtain waveforms of ROI-wise activity (see below).

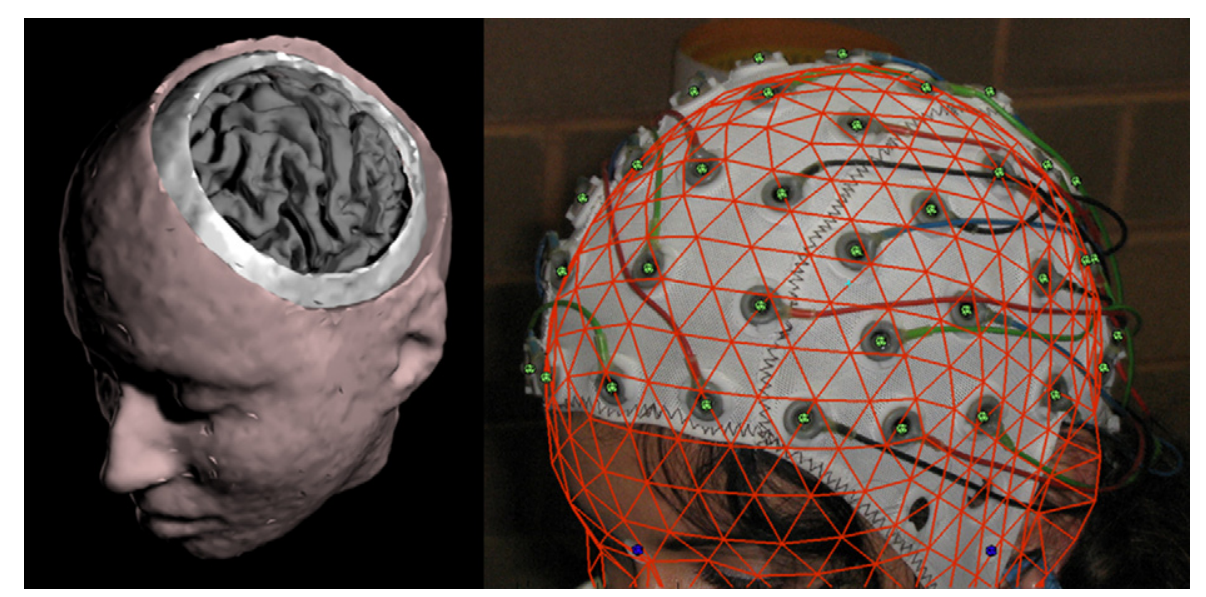

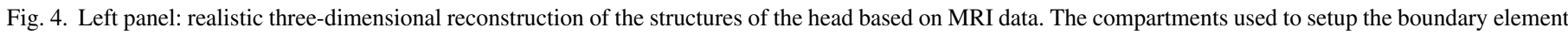

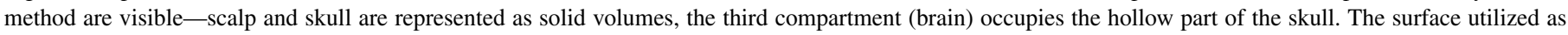

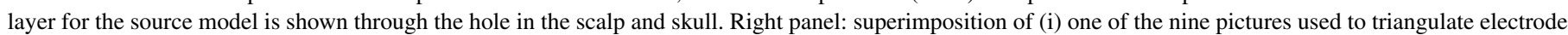

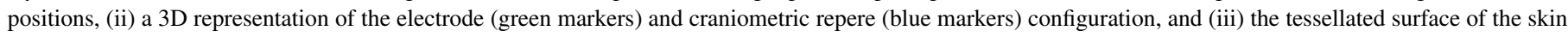
used in the BEM. The point of view of the $3 \mathrm{D}$ reconstructions is set according to camera position output by the phototriangulation procedure.

Please cite this article in press as: Cincotti F, et al., High-resolution EEG techniques for brain-computer interface applications, J Neurosci Methods (2007), doi:10.1016/j.jneumeth.2007.06.031 


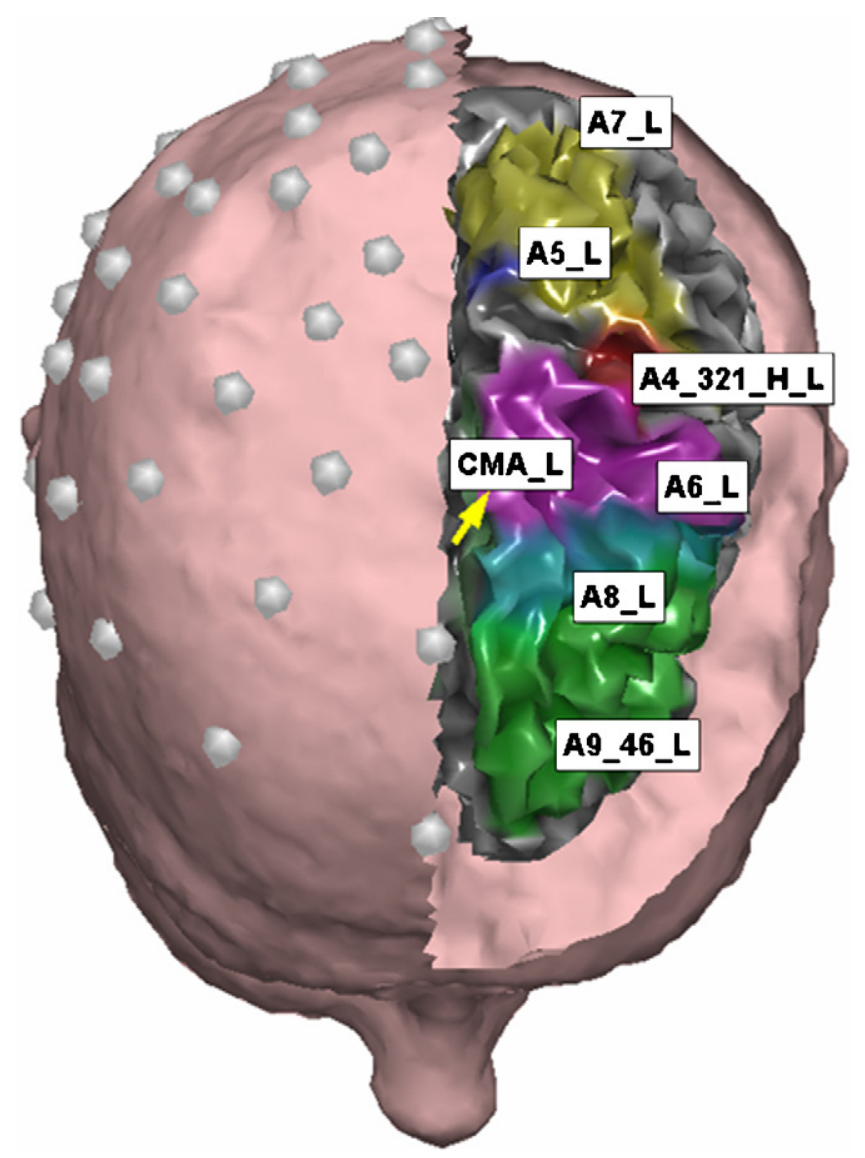

Fig. 5. Regions of interest (ROIs), depicted in colors for the on-line version of the paper, or in a grayscale on the printed journal, on a realistic model of the head for a particular subject involved in the experiments. Labels indicate the name of the ROIs, with the postfix L or R for left and right. For instance, the ROI indicated with the label (A8_L) is the Brodmann area 8 left.

Fig. 5 illustrates a set of the ROIs on a realistic cortical reconstruction, obtained in a given experimental subject.

\subsection{Determination of electrode positions}

The position of electrodes was measured trough photogrammetric techniques. Using a commercial computer program (Photomodeler 5, Eos Systems Inc., Vancouver, Canada) a consumer digital camera was calibrated. Before any acquisition session, as soon as the electrode cap was positioned on the subject's head, 9 pictures of the electrode montage were taken from different angles covering the whole head. Three craniometric repere points (nasion, left and right preauricular points) were marked on the subject's skin to allow later registration to the BEM head model. Within the Photomodeler program, electrodes were manually marked on each picture, and references between markers of the same electrode on different picture were made. Triangulation between such markers yielded a 3D model of each electrode, together with the reconstruction of the camera position and orientation for each shot. Since triangulation procedures cannot compute the actual scale factor, this was fixed at a later stage.
An affine, isotropic transformation was performed to align the craniometric points in the same reference system used for the BEM model, and to scale the distance of preauricular points to the measure made on the BEM model. Fig. 2, right panel, shows a representation of the registration procedure.

\subsection{Estimation of cortical source current density}

The solution of the neuroelectrical linear inverse problem can be factorized into the matrix multiplication of a pseudoinverse matrix $(\mathbf{G})$ times the vector of measured potentials. This factorization allows to pre-compute $\mathrm{G}$, as soon as the geometry of the model (BEM head model, source model and sensor positions) is available. Matrix multiplication is a very fast procedure, and estimation of source estimates can thus be performed on large datasets (unaveraged EEG) and even in real-time.

Computation of the G matrix was implemented in Matlab 7.3 (The Mathworks, Natick, MA). A description of the algorithms is given elsewhere, together with simulations that validate the procedure (Babiloni et al., 2000, 2005).

The solution of the following linear system:

$\mathbf{A x}=\mathbf{b}+\mathbf{n}$

provides an estimation of the dipole source configuration $\mathbf{x}$ that generates the measured EEG potential distribution $\mathbf{b}$. The system includes also the measurement noise $\mathbf{n}$, assumed to be normally distributed (Dale and Sereno, 1993; Grave de Peralta Menendez and Gonzalez Andino, 1999; Nunez, 1995). A is the lead field matrix, where each $j$-th column describes the potential distribution generated on the scalp electrodes by the $j$-th unitary dipole. The current density solution vector $\xi$ of Eq. (1) was obtained as (Grave de Peralta Menendez and Gonzalez Andino, 1999):

$\xi=\underset{x}{\arg \min }\left(\|\mathbf{A} x-b\|_{\mathbf{M}}^{2}+\lambda^{2}\|x\|_{\mathbf{N}}^{2}\right)$

where $\mathbf{M}, \mathbf{N}$ are the matrices associated to the metrics of the data and of the source space, respectively, $\lambda$ the regularization parameter and $\|x\|_{M}$ represents the $\mathrm{M}$ norm of the vector $\mathbf{x}$. The solution of Eq. (2) is given by the inverse operator $\mathbf{G}$ :

$\xi(t)=\mathbf{G b}(t), \quad \mathbf{G}=\mathbf{N}^{-1} \mathbf{A}^{\prime}\left(\mathbf{A} \mathbf{N}^{-1} \mathbf{A}^{\prime}+\lambda \mathbf{M}^{-1}\right)^{-1}$

An optimal regularization of this linear system was obtained by the L-curve approach (Hansen, 1992a,b). As a metric in the data space we used the identity matrix, while as a norm in the source space we use the following metric:

$\left(\mathbf{N}^{-1}\right)_{i i}=\left\|\mathbf{A}_{. \mathbf{i}}\right\|^{-2}$

where $\left(\mathbf{N}^{-1}\right)_{i i}$ is the $i$-th element of the inverse of the diagonal matrix $\mathbf{N}$ and all the other matrix elements $\mathbf{N}_{i j}$ are set to 0 . The $\mathrm{L}_{2}$ norm of the $i$-th column of the lead field matrix $\mathbf{A}$ is denoted by $\left\|\mathbf{A}_{\mathbf{i}}\right\|$.

Using the relations described above, an estimate of the signed magnitude of the dipolar moment for each cortical dipoles was obtained for each time point. As the orientation of the dipole was defined to be perpendicular to the local cortical surface in the head model, the estimation process returned a scalar rather than 
a vector field. The spatial average of the signed magnitude of all the dipoles belonging to a particular ROI at each time sample was used to estimate the waveforms of cortical ROI activity in that ROI $(\rho(t))$.

Spatial averaging can be expressed in terms of matrix multiplication by a matrix T. This matrix is sparse and has as many rows as ROIs and as many columns as the number of dipole sources. ROI CCD waveforms can then be expressed as:

$\rho(t)=\mathbf{T} \boldsymbol{\chi}(t)=\mathbf{T G b}(t)=\mathbf{G}_{\mathrm{ROI}} \mathbf{b}(t), \quad \mathbf{G}_{\mathrm{ROI}}=\mathbf{T G}$

The $\mathbf{G}_{\mathrm{ROI}}$ matrix only depends on geometrical factors, and can thus be computed and stored off-line. The matrix multiplication in Eq. (4) can be interpreted as a spatial filtering of the scalp potenital $\mathbf{b}(t)$, using the elements of $\mathbf{G}_{\mathrm{ROI}}$ as weights. Thus, it was possible to utilize the results of CCD linear estimate in the BCI framework by plugging the weights derived here into the spatial filtering block of the BCI system, both off-line and on-line.

\section{Results}

Fig. 6 shows the typical cortical current density waveforms obtainable from the mathematical procedure described in Eqs. (1)-(4). In particular, it can be appreciated the particular region of interests (ROIs) representing the primary motor area for the hand and the lips movement, depicted in red and blue, respectively, on the realistic cortical model. The averaged CCD activities within the ROIs during the time period of a particular task is represented as waveforms on the right of the Figure. The task requires also the lip pursuing together the hand movement imagination. The waveforms at the bottom of the figure are relative to the scalp potentials gathered from the electrodes $\mathrm{C} 3$ and C4. Fig. 6 (top panel) was obtained by processing the single trial of the recorded EEG, and by applying the procedure for the estimation of the CCD waveforms described in the Methods section. It can be possible appreciate the time resolution still available at the level of the cortical ROIs considered.

In the bottom panel of Fig. 6, the topographical distribution of $r^{2}$ values in the alpha band are represented for subject 3 . Raw EEG (left side) yields a wide distribution over the bilateral central regions of the scalp, and has a peak value around 0.3. Estimated CCD (right side) produces more localized regions of relevant activity, with a higher (0.6) peak value.

By applying the above mentioned signal processing techniques in the context of the proposed BCI setup, we used the $r^{2}$ as an index of reliability of the recognition of subject's mental activity. The comparisons between the maximum values of the $r^{2}$ that takes into account the best usable feature (frequency/ROI or scalp channel) were performed for the unprocessed EEG data as well as for the estimated cortical activity by using the procedure already described above. As shown in Fig. 7, the values for the $r^{2}$ index are always larger than those obtained for the same subject by using the raw scalp potentials during the execution of the task. Mean $r^{2}$ is $0.20 \pm 0.114$ S.D. for the RAW case, $0.55 \pm 0.16$ S.D. for the CCD case. The differences are relatively constant across the subjects, and a paired Student's $t$-test returned a highly significant differences between the two conditions $\left(p<10^{-5}\right)$.

The results provided above suggest the possibility to use efficiently the proposed approach to improve recognition rates of the brain-computer interfaces based on non invasive EEG recordings. To support such conclusions, we also applied the cortical imaging technique to a more challenging experimental situation. Two independent control signals were derived by combination of EEG features, so that both coordinates the feedback cursor could be controlled by the subject. Eight targets were distributed against the screen sides. From an starting point located in the center of the screen, the subject had to move the cursor towards the highlighted target. The cortical waveforms obtained by the application of the linear inverse procedure are processed on-line. This is possible since the computational burden for such procedure is not elevate, due to the matrix multiplication required to compute the estimated cortical current density, as described by Eq. (3), due to the storage of the pseudoinverse matrix $\mathrm{G}$ on the hard disk of the computer. Preliminary results of the use of the on-line CCD estimation reports that one subject is able to control the cursor direction in a plane, with a rate of correct hits about the $80 \%$, compared to $71 \%$ obtained with the use of the raw EEG potential. It is worth noting that in this experimental condition, the chance level is at $12.5 \%$ (well below the $50 \%$ level of a binary task). Fig. 8 shows the subject involved in the task of control the direction of the red cursor, at the center of the screen, in order to hit in a predefined time-frame the target (depicted as a red bar on the right of the screen).

\section{Discussion}

The attempt to recognize patterns from the EEG potentials is at the base of the field of the brain-computer interface (Cincotti et al., 2002, 2003; Millán et al., 2002, 2003, 2004; Wolpaw and McFarland, 2004; Wolpaw et al., 1991, 2002). Processing EEG signals mainly involves two steps: (1) the relevant EEG features must be extracted from raw signals. (2) These features must be effectively transformed (classified) so that the output control signal (command) is as close as possible to the user's will.

In this paper we proposed a procedure for the estimation of the cortical waveforms from the recorded EEG potentials in the framework of the brain-computer interface, and its use on an on-line in a BCI system. The results obtained from a pilot study on a population of normal subjects trained to operate a BCI based on modulation of sensorimotor EEG rhythms suggest that the use of CCD is superior to the use of scalp EEG potentials for off-line classification of mental imagery. Further research is necessary to extend to other BCI systems the results obtained in this experiments.

Since the present analysis approach is based on the use of a cortical model as a source space for the estimated cortical activity from the scalp EEG recordings, it may be asked what happens if deep brain sources are active during the execution of the experimental task. EEG activity at the scalp are generally considered to be generated mainly by cortical sources because of the columnar arrangement of cortical neurons (Nunez, 1995). Like other source models that neglect the contribution of "other sources" 


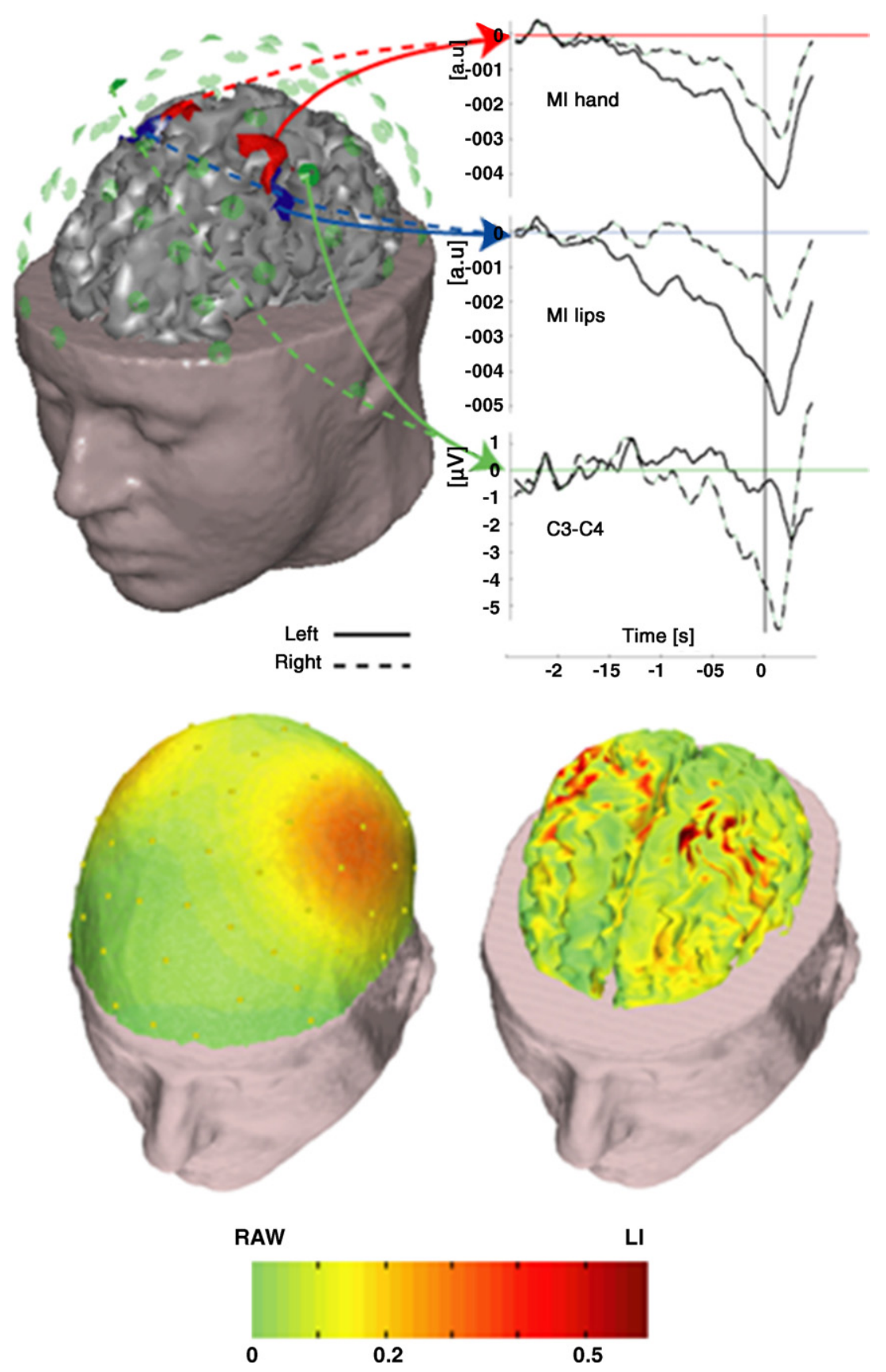

Fig. 6. Top panel: cortical current density (CCD) waveforms estimated in two regions of interest (ROIs) coincident with the primary motor area for the hand movement (in red for the on-line version and in light gray for the printed version) and for the lip movement (in blue for the on-line version and in dark gray for the printed version) on a realistic representation of the subject's head. The bullets are the 3D representation of the scalp electrodes employed for the EEG recordings. Bottom panel: maps of statistical significance of all recorded EEG channels (left) and modeled cortical sources (right).

(e.g., dipolar models), the cortical current density source model has been shown by a number of investigators to be a valid source model in which the weak contribution of deeper sources is explicitly neglected. In many cases, deeper sources produce little external field that could generally be neglected in a given source model dealing with cortical dynamics, although this is not as true as in the case of hypothetical so-called closed fields that in principle would produce no measurement at all. Therefore it is reasonable to assume that the source space for the present inverse problem lies in principle on the cortical mantle of the brain. The use of the realistic head models to estimate the CCD on the cortical mantle was performed by using a 3-shell BEM. Although other different methods could return an estimate of cortical activity from superficial EEG recordings (i.e., finite element modeling, FEM) we choose the BEM for the generation of the lead field matrix due to stability of the results that such approach provides. In fact, the potential improvements provided by the finite element model approach (FEM), that is able to model precisely the different structures within the head, are frustrated by the lack of precise values for tissue conductivity

Please cite this article in press as: Cincotti F, et al., High-resolution EEG techniques for brain-computer interface applications, J Neurosci Methods (2007), doi:10.1016/j.jneumeth.2007.06.031 


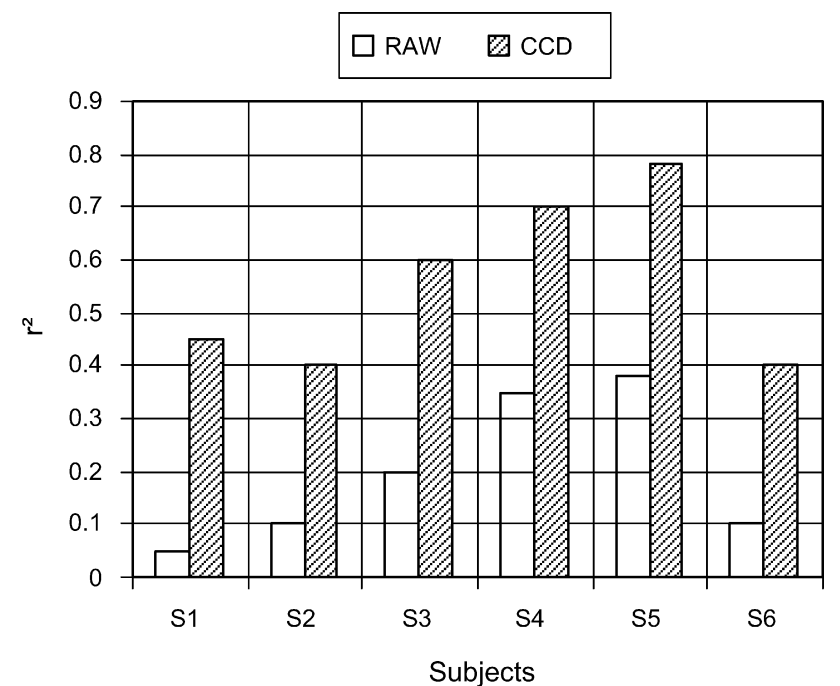

Fig. 7. Figure shows the values of the $r^{2}$ index for the best features extracted from the scalp recorded EEG (RAW) and from the CCD waveforms (CCD) in six subjects during the execution of the task related to the cursor control. The $r^{2}$ generated for the CCD waveforms are always above the values obtained by using the raw EEG potentials, with an high statistical significance.

(which should be modeled as a inhomogeneous and anisotropic tensor field). In addition, the use of 3-shell BEM approach for the head models adopted here has been demonstrated superior to the use of 1-shell BEM approach, In fact such approach was suitable in presence of magnetoencephalographic measurements due to the fact of the relative insensitivity of the magnetic field to the different conductivity values relative to the different skull, scalp and brain tissues. Instead, the differences in conductivity between such head structures have a strong impact on the estimation of the CCD with the use of EEG recordings (Fuchs et al., 2007). It is also worth of note that the differences in esti-

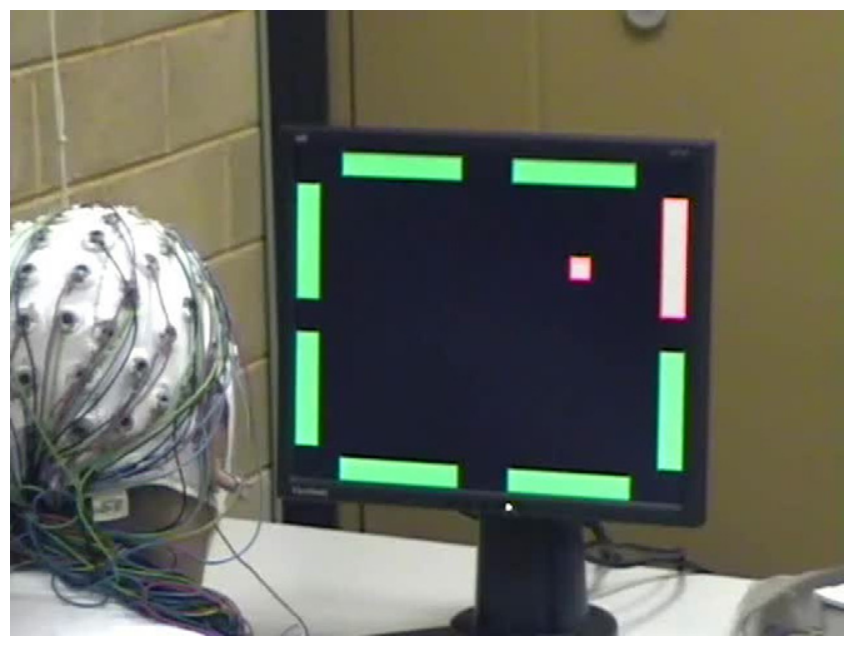

Fig. 8. Figure shows a subject involved in the task of control the direction of the cursor, at the center of the screen, in order to hit in a predefined time-frame the target (depicted as a red bar in the on-line version of the paper and in light gray on the printed version on the right of the screen). The task was accomplished by using on-line the estimation of the cortical current density waveforms. mated CCD due to the use of modeling the cerebro-spinal fluid $(\mathrm{CSF})$ in a 4-shell BEM instead of a 3-shell BEM approach are negligible here (Fuchs et al., 2007; Michel et al., 2004).

However, mapping the pattern of CCD on the subject's cortex is only a partial result. Indeed, solving the inverse problem allows determining the electrical activity of every small area of the cortex, i.e., in the way that can be represented by a map. Nevertheless, given its high temporal resolution, the time course of representative regions of the brain should be visualized. Moreover, inter-subject statistics should be carried on, meaning that a matching should be found between corresponding regions of different brain, according to the individual anatomical distribution of the considered cortical functions. Finally, intra-subject and inter-subject statistics need an appropriate transformation to normalize CCDs.

The first instrument in this direction is the segmentation of individual areas corresponding to the specific functions. The segmentation of these regions of interest (ROIs) can be performed a posteriori, by selecting the significant "clusters" of two-dimensional (i.e., cortically constrained) activity mapping, reasonably corresponding to the functional responsive areas. Alternatively, the selection of an activity "cluster" can be performed a priori, either using the cortical folds as a reference or taking advantage from the normalized position of equivalent sources derived from a Talairach brain transformation. As far as the ROIs cover a small patch of cortex, the average CCD on each of the ROIs can be taken as a correlate of the activity of a functional area, and its time course can be plotted as a waveform. Most of the analyses that have been developed for scalp potentials (averaging, spectral analysis, coherence, etc.) can be applied to CCD waveforms, empowering the capacity of these analyses. The present results were obtained by the application of the functional connectivity estimator to the high-density EEG data in the frequency domain. Frequencies up to $45 \mathrm{~Hz}$ were investigated, although recently the presence of important information at higher ranges of temporal frequencies have been suggested (Gonzalez et al., 2006). However, the results of the off-line and on-line experimentation suggest that the approach to use CCD waveforms to control a BCI system based on voluntary modulation of sensorimotor EEG rhythms are encouraging and highly statistically significant.

Despite complexity of the mathematics involved, on-line application was kept as simple as possible. The proposed procedure was designed to be implemented with minimal impact into the signal pre-processing stage of a BCI, in place of any existing instantaneous linear combination (i.e., spatial filtering) block. With the particular implementation of BCI used in this study (BCI2000), this was achieved simply by loading the $\mathbf{G}_{\mathrm{ROI}}$ matrix from the user interface. Given the wide diffusion of this sofware in different research laboratories, these results are also interesting in the perspective to apply such technology in a larger experimental group, possibly involving the person with motor disabilities.

In fact, in the field of rehabilitation, the main goal is the reduction of the disability provoked by any pathological condition that is the achievement of the maximum independence for a given clinical frame. 
The key issues in this research context will be the development of a system that allows a seamless interaction between the user and with a wide variety of actuators (robots, domotic appliances), independently of the kind of input device (aid) the user utilizes. The implementation of this system will take into account those stages of the disease in which the residual muscular strength could be not adequate, if present, for the utilization of conventional aids and in those conditions in which practical obstacles or security concerns could prevent a displacement from bed.

The ultimate product will be an integrated communicationcontrol system, customized on the residual abilities of each severely motor impaired patient,. Clinical validation of the device will provide for the documentation about patients feedback and guidelines for future development of a device to be deployed to the patients'houses.

\section{Acknowledgements}

This work has been partially supported by the Italian Telethon Foundation (Grant: GUP03562), by the support of the Minister for Foreign Affairs, Division for the Scientific and Technologic Development, in the framework of a bilateral project between Italy and China (Tsinghua University), the support of the European Union, through the MAIA project (IST Programme FET Project FP6-003758), by the EU COST Project NEUROMATH (BM0601) and was supported in part by a grant from NIH (EB006356) in the USA. This paper only reflects the authors' views and funding agencies are not liable for any use that may be made of the information contained herein.

\section{References}

Babiloni F, Babiloni C, Locche L, Cincotti F, Rossini PM, Carducci F. High-resolution electro-encephalogram: source estimates of Laplaciantransformed somatosensory-evoked potentials using a realistic subject head model constructed from magnetic resonance images. Med Biol Eng Comput 2000;38(5):512-9.

Babiloni C, Babiloni F, Carducci F, Cincotti F, Cocozza G, Del Percio C, et al. Human cortical EEG rhythms during the observation of simple aimless movements. A high resolution EEG study. Neuroimage 2002;17(2):559-72.

Babiloni C, Babiloni F, Carducci F, Cappa SF, Cincotti F, Del Percio C, et al. Human cortical responses during one-bit short-term memory. A high-resolution EEG study on delayed choice reaction time tasks. Clin Neurophysiol 2004a;115(1):161-70.

Babiloni C, Miniussi C, Babiloni F, Carducci F, Cincotti F, Del Percio C, et al. Sub-second "temporal attention" modulates alpha rhythms. A highresolution EEG study. Cogn Brain Res 2004b;19(3):259-68.

Babiloni F, Cincotti F, Babiloni C, Carducci F, Basilisco A, Rossini PM, et al. Estimation of the cortical functional connectivity with the multimodal integration of high resolution EEG and fMRI data by directed transfer function. Neuroimage 2005;24(1):118-31.

Birbaumer N, Cohen LG. Brain-computer interfaces (BCI): communication and restoration of movement in paralysis. J Physiol 2007;579(Pt 3):621-36.

Cincotti F, Mattia D, Babiloni C, Carducci F, Bianchi L, del RM, et al. Classification of EEG mental patterns by using two scalp electrodes and Mahalanobis distance-based classifiers. Methods Inf Med 2002;41(4):337-41.

Cincotti F, Mattia D, Babiloni C, Carducci F, Salinari S, Bianchi L, et al. The use of EEG modifications due to motor imagery for brain-computer interfaces. IEEE Trans Neural Syst Rehabil Eng 2003;11(2):131-3.
Cincotti F, Babiloni C, Miniussi C, Carducci F, Moretti D, Salinari S, et al. EEG deblurring techniques in a clinical context. Methods Inf Med 2004a;43(1):114-7.

Dale AM, Sereno M. Improved localization of cortical activity by combining EEG and MEG with MRI cortical surface recostruction: a linear approach. J Cogn Neurosci 1993;5:162-76.

Dale AM, Liu AK, Fischl BR, Buckner RL, Belliveau JW, Lewine JD, et al. Dynamic statistical parametric mapping: combining fMRI and MEG for high-resolution imaging of cortical activity. Neuron 2000;26(1):5567.

Fuchs M, Wagner M, Kastner J. Development of volume conductor and source models to localize epileptic foci. J Clin Neurophysiol 2007;24(2): 101-19.

Gonzalez SL, Grave de Peralta R, Thut G, Millan J, del R, Morier P, et al. Very high frequency oscillations (VHFO) as a predictor of movement intentions. Neuroimage 2006;32:170-9.

Grave de Peralta R, Gonzalez Andino SL, Morand S, Michel CM, Landis T. Imaging the electrical activity of the brain: ELECTRA. Hum Brain Map 2000;9:1-12.

Grave de Peralta Menendez R, Gonzalez Andino SL. Distributed source models: standard solutions and new developments. In: Uhl C, editor. Analysis of neurophysiological brain functioning. Springer Verlag; 1999. p. 176201.

Hämäläinen M, Ilmoniemi R. Measured magnetic field of the brain: estimates of the current distributions. Technical report TKK-F-A559. Helsinki: Helsinki University of Technology, 1984.

Hansen PC. Analysis of discrete ill-posed problems by means of the L-curve. SIAM Rev 1992a;34:561-80.

Hansen PC. Numerical tools for the analysis and solution of Fredholm integral equations of the first kind. Inverse Probl 1992b;8:849-72.

He B, Hori J, Babiloni F. EEG inverse problems. In: Akay M, editor. Wiley encyclopedia in biomedical engineering, vol. 2. John Wiley \& Sons, Inc.; 2006. p. $1355-63$

Mattia D, Cincotti F, Mattiocco M, Scivoletto G, Marciani MG, Babiloni F. Motor-related cortical dynamics to intact movements in tetraplegics as revealed by high-resolution EEG. Hum Brain Map 2006;27(6): 510-9.

McFarland DJ, McCane LM, David SV, Wolpaw JR. Spatial filter selection for EEG-based communication. Electroencephalogr Clin Neurophysiol 1997;103(3):386-94.

Michel CM, Murray MM, Lantz G, Gonzalez S, Spinelli L, Grave DP. EEG source imaging. Clin Neurophysiol 2004;115(10):2195-222.

Millán J, Mouriño J, Franzé M, Cincotti F, Varsta M, Heikkonen J, et al. A local neural classifier for the recognition of EEG patterns associated to mental tasks. IEEE Trans Neural Netw 2002;13(3):678-86.

Millán J, del R, Mouriño J. Asynchronous BCI and local neural classifiers: an overview of the adaptive brain interface project. IEEE Trans Neural Syst Rehabil Eng 2003;11(2):159-61.

Millán J, del R, Renkens F, Mouriño J, Gerstner W. Non-invasive brainactuated control of a mobile robot by human EEG. IEEE Trans Biomed Eng 2004;51(6):1026-33.

Nunez PL. Neocortical dynamics and human EEG rhythms. Oxford University Press; 1995

Oostendorp TF, Delbeke J, Stegeman DF. The conductivity of the human skull: results of in vivo and in vitro measurements. IEEE Trans Biomed Eng 2000;47(11):1487-92.

Picard N, Strick PL. Imaging the premotor areas. Curr Opin Neurobiol 1996;11(6):663-72.

Schalk G, McFarland DJ, Hinterberger T, Birbaumer N, Wolpaw JR. BCI2000: a general-purpose brain-computer interface (BCI) system. IEEE Trans Biomed Eng 2004;51(6):1034-104.

Urbano A, Babiloni C, Onorati P, Ambrosini A, Carducci F, Fattorini L, et al. Responses of human primary sensorimotor and supplementary motor areas to internally-triggered unilateral and simultaneous bilateral one-digit movements. A high resolution EEG study. Eur J Neurosci, Cambridge, UK 1998;10(2):765-70

Wolpaw JR. Brain-computer interfaces as new brain output pathways. J Physiol 2007;579(Pt 3):613-9. 
Wolpaw JR, Birbaumer N. Brain-computer interfaces for communication and control. In: Selzer ME, Clarke S, Cohen LG, Duncan P, Gage FH, editors. Textbook of neural repair and rehabilitation, neural repair and plasticity. Cambridge: Cambridge University Press; 2006. p. 602-14.

Wolpaw JR, McFarland D. Control of a two-dimensional movement signal by a noninvasive brain-computer interface in humans. Proc Natl Acad Sci USA 2004;101(51):17849-54.
Wolpaw JR, McFarland DJ, Neat GW, Forneris CA. An EEG-based braincomputer interface for cursor control. Electroencephalogr Clin Neurophysiol 1991;78(3):252-9.

Wolpaw JR, Birbaumer N, McFarland DJ, Pfurtscheller G, Vaughan TM. Braincomputer interfaces for communication and control. Clin Neurophysiol 2002;113(6):767-91.

Please cite this article in press as: Cincotti F, et al., High-resolution EEG techniques for brain-computer interface applications, J Neurosci Methods (2007), doi:10.1016/j.jneumeth.2007.06.031 